\title{
Foot segments mobility and plantar pressure in the normal foot
}

\author{
Paolo Caravaggi $i^{*}$, Claudia Giacomozzi ${ }^{2}$, Alberto Leardini ${ }^{1}$ \\ From 4th Congress of the International Foot and Ankle Biomechanics (i-FAB) Community \\ Busan, Korea. 8-11 April 2014
}

\section{Background}

The foot is generally regarded as a flexible structure which can adjust its flexibility in response to variable dynamic conditions in different phases within different motor tasks. In gait, both kinematics and baropodometry have shown to be affected by functional and structural factors [1]. In fact pressure distribution can be seen as the effectiveness of the musculoskeletal system in absorbing the ground reaction forces via the foot and its joints. Excessive foot pressure may develop into calluses, which become sites of peak pressure and pain. The relationship between foot joints mobility and plantar pressure has not been thoroughly investigated. Aim of this study was to combine a multi-segment kinematics model [2] and baropodometric analysis based on anatomical masking [3], to investigate correlations between intersegmental kinematics and regional baropodometric parameters in the normal foot.

\section{Materials and methods}

Ten able-bodied subjects ( $26.8 \pm 6.9$ years; $67.5 \pm 12.6 \mathrm{Kg})$ volunteered in the study. An eight-camera motion system (Vicon, UK) was used to track foot segments during the stance phase of level walking, according to an established protocol (Figure 1, top) [2]. Simultaneously, a pressure plate (Novel, Gmbh) recorded foot plantar pressure over three repetitions. An anatomical-based selection of areas of interest was employed to divide the pressure footprints in seven subareas (Figure 1, bottom) [3]. Maximum of mean and peak pressure, of vertical force, contact-area and -time, and pressure- / force-time integrals, were determined for each subarea. The relationship between range of

'Movement Analysis Laboratory, Istituto Ortopedico Rizzoli, Bologna, 40136, Italy

Full list of author information is available at the end of the article

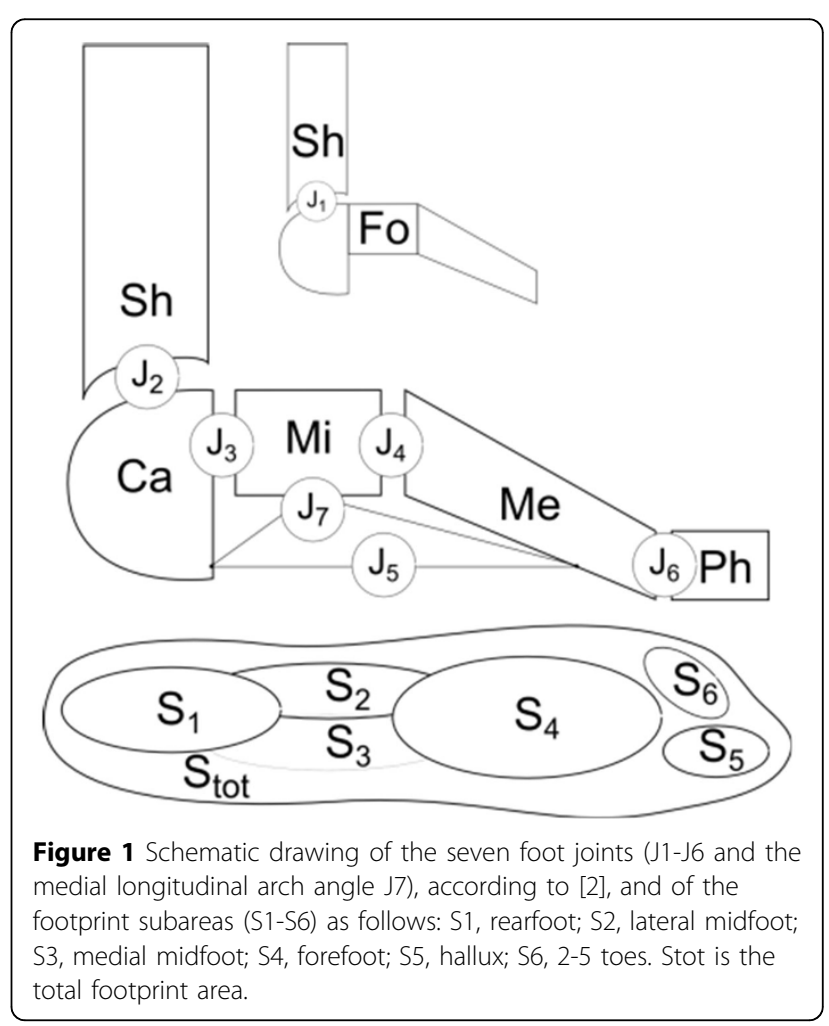

motion (ROM) of each foot joint and baropodometric parameters in each subarea was investigated using Pearson's and Spearman's coefficients.

\section{Results}

Most of the statistically significant correlations $(\mathrm{p}<0.05)$ between foot joints ROM and baropodometric parameters were moderate $(|R|=0.36-0.67)$. In general, mean and peak pressure at rearfoot and forefoot were negatively correlated with the amount of motion at the 


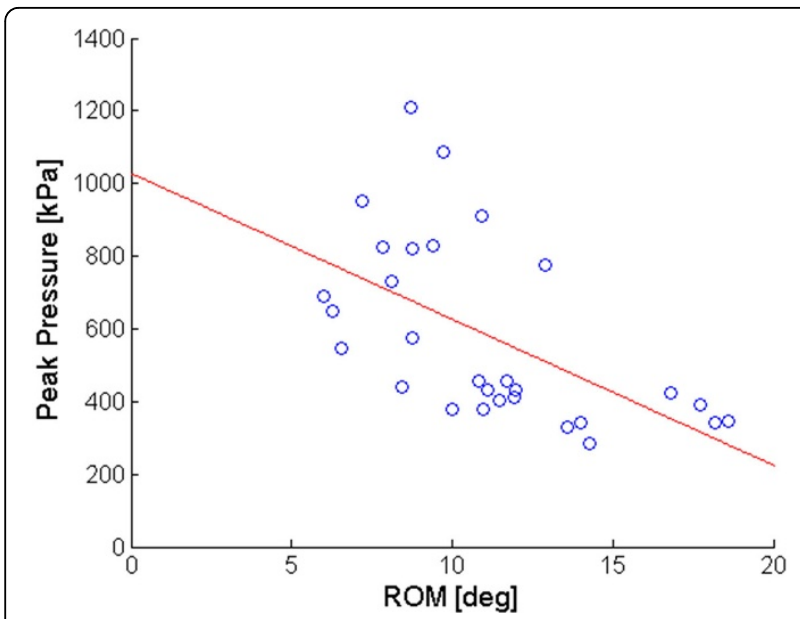

Figure 2 Scatter plot of the relationship between peak pressure $(\mathrm{kPa})$ at the forefoot ( $\mathrm{S} 4$ in fig. 1) and sagittal-plane ROM (deg) at the tarso-metatarsal joint (J4 in fig.1). The linear regression line is superimposed to the data points.

ankle and tarso-metatarsal joints (Figure 2). In contrast, pressure at the hallux and midfoot were positively correlated with the ROM of the joints across the midfoot. Strong correlation was found between ROM of the medial longitudinal arch angle (J7) and pressure-time-integral at the forefoot (Spearman Rho $=-0.93, \mathrm{p}<0.05)$.

\section{Conclusions}

According to the sample of normal feet analyzed in this study, those feet presenting smaller joint mobility are associated with larger pressure at the rear- and forefoot. A trend for decreased pressure at the midfoot and toes was also detected in feet with a stiffer medial longitudinal arch.

\section{Authors' details}

${ }^{1}$ Movement Analysis Laboratory, Istituto Ortopedico Rizzoli, Bologna, 40136, Italy. ${ }^{2}$ Department of Technology and Health, Istituto Superiore di Sanità,

Roma, 00161, Italy.

Published: 8 April 2014

\section{References}

1. Morag E, Cavanagh PR: Structural and functional predictors of regional peak pressures under the foot during walking. J Biomechanics 1997, 32:359-370.

2. Leardini $A$, et al: Rear-foot, mid-foot and fore-foot motion during the stance phase of gait. Gait and Posture 2007, 25:453-62.

3. Giacomozzi C, et al: Distributed loading and multi-segment kinematics for the functional evaluation of foot pathologies: preliminary experience with varying degrees of flat-foot severity. Proceedings of the XXIV Congress of ISB Natal, Brazil; 2013, 75.

doi:10.1186/1757-1146-7-S1-A11

Cite this article as: Caravaggi et al:: Foot segments mobility and plantar pressure in the normal foot. Journal of Foot and Ankle Research 2014 7(Suppl 1):A11.

\section{Submit your next manuscript to BioMed Central} and take full advantage of:

- Convenient online submission

- Thorough peer review

- No space constraints or color figure charges

- Immediate publication on acceptance

- Inclusion in PubMed, CAS, Scopus and Google Scholar

- Research which is freely available for redistribution 